Submitted to the Annals of Statistics

\title{
SUPPLEMENT TO COUPLING METHODS FOR MULTISTAGE SAMPLING
}

\author{
By Guillaume Chauvet \\ CREST-ENSAI*
}

In this Supplement, we provide additional proofs in Section 1. A technical lemma used for Theorem 3.1 is proved in Section 1.1. The proofs of Propositions 3.1 and 3.2 are given in Section 1.2 and 1.3, respectively. The proof of Proposition 4.1 is given in Section 1.4, along with the proof of two technical lemmas. The proof of Propositions 4.2, 4.3 and 4.6 are given in Sections 1.5, 1.6 and 1.7, respectively. Additional simulation results are presented in Section 2.

\section{Additional Proofs.}

1.1. Additional results for Theorem 3.1.

Lemma 1.1. Assume that (H1) holds. Then

$$
\begin{array}{r}
\left(1-f_{I}\right)^{N_{I}} \underset{t \rightarrow \infty}{\longrightarrow} 0 \\
\sum_{k=1}^{N_{I}} \frac{C_{N_{I}}^{k} f_{I}^{k}\left(1-f_{I}\right)^{N_{I}-k}}{k} \underset{t \rightarrow \infty}{\longrightarrow} 0 .
\end{array}
$$

Proof . Equation (A1) follows immediately. Consider now the truncated binomial random variable $X$ with parameters $N_{I}$ and $f_{I}$, defined for $k=1, \ldots, N_{I}$ by $\operatorname{Pr}(X=k)=\omega C_{N_{I}}^{k} f_{I}^{k}\left(1-f_{I}\right)^{N_{I}-k}$ with $\omega=\frac{1}{1-\left(1-f_{I}\right)^{N_{I}}}$. Then from inequality (27) in [2]

$$
\sum_{k=1}^{N_{I}} \frac{C_{N_{I}}^{k} f_{I}^{k}\left(1-f_{I}\right)^{N_{I}-k}}{k}=\frac{1}{\omega} E\left(\frac{1}{X}\right) \leq \frac{1}{\omega}\left\{1-\frac{\left(n_{I} \omega-1\right)^{2}}{N_{I}\left(N_{I}-1\right) f_{I}^{2} w}\right\}
$$

and (A2) follows from (A1) and assumption (H1).

1.2. Proof of Proposition 3.1. Since $E\left(\Delta_{2}\right)=0$, we have $E\left\{\left(\Delta_{2}\right)^{2}\right\}=$ $V\left(\Delta_{2}\right)$. Also, we can write $\Delta_{2}=\epsilon \sum_{u_{i} \in S_{I}^{+}}\left(\hat{Y}_{i}-\mu_{Y}\right)$ where $\epsilon=1$ if $n_{I}^{B}<n_{I}$ and $\epsilon=-1$ otherwise. We have

$$
V\left(\Delta_{2}\right)=E V_{\left\{S_{I}^{B}, S_{I}\right\}}\left(\Delta_{2}\right)+V E_{\left\{S_{I}^{B}, S_{I}\right\}}\left(\Delta_{2}\right) .
$$

imsart-aos ver. 2014/10/16 file: SupplementaryMaterial_Rev2.tex date: May 17, 2015 
Using the independence in the second-stage selections and the fact that $S_{I}^{+} \sim S I\left(U_{I} ;\left|n_{I}^{B}-n_{I}\right|\right)$, we obtain

$$
\begin{aligned}
E V_{\left\{S_{I}^{B}, S_{I}\right\}}\left(\Delta_{2}\right) & =E\left|n_{I}^{B}-n_{I}\right| N_{I}^{-1} \sum_{u_{i} \in U_{I}} V_{i}, \\
V E_{\left\{S_{I}^{B}, S_{I}\right\}}\left(\Delta_{2}\right) & \leq E\left|n_{I}^{B}-n_{I}\right| N_{I}^{-1} \sum_{u_{i} \in U_{I}}\left(Y_{i}-\mu_{Y}\right)^{2} .
\end{aligned}
$$

By plugging (A4) and (A5) into (A3), and using the inequality

$$
E\left|n_{I}^{B}-n_{I}\right| \leq\left\{V\left(n_{I}^{B}\right)\right\}^{0.5}=\left\{n_{I}\left(1-f_{I}\right)\right\}^{0.5},
$$

we obtain

$$
V\left(\Delta_{2}\right) \leq\left\{n_{I}\left(1-f_{I}\right)\right\}^{0.5}\left\{N_{I}^{-1} \sum_{u_{i} \in U_{I}}\left(Y_{i}-\mu_{Y}\right)^{2}+N_{I}^{-1} \sum_{u_{i} \in U_{I}} V_{i}\right\} .
$$

Adapting equation (3.2), we have

$$
\begin{aligned}
V\left\{\sum_{u_{i} \in S_{I}^{B}}\left(\hat{Y}_{i}-\mu_{Y}\right)\right\} & =n_{I}\left\{\left(1-f_{I}\right) N_{I}^{-1} \sum_{u_{i} \in U_{I}}\left(Y_{i}-\mu_{Y}\right)^{2}+N_{I}^{-1} \sum_{u_{i} \in U_{I}} V_{i}\right\} \\
& \geq n_{I}\left(1-f_{I}\right)\left\{N_{I}^{-1} \sum_{u_{i} \in U_{I}}\left(Y_{i}-\mu_{Y}\right)^{2}+N_{I}^{-1} \sum_{u_{i} \in U_{I}} V_{i}\right\}
\end{aligned}
$$

so that Proposition 3.1 follows.

1.3. Proof of Proposition 3.2. We can write $\Delta_{3} \equiv N_{I}^{-2} n_{I}\{v(\hat{Y})-V(\hat{Y})\}=$ $T_{3}+T_{4}$, with

$$
\begin{aligned}
& T_{3}=\frac{1}{N_{I}} \sum_{u_{i} \in S_{I}} \hat{V}_{i}-\frac{f_{I}}{N_{I}} \sum_{u_{i} \in U_{I}} V_{i}, \\
& T_{4}=\left(1-f_{I}\right) s_{Z}^{2}-\left(1-f_{I}\right)\left\{S_{Y, U_{I}}^{2}+\frac{1}{N_{I}} \sum_{u_{i} \in U_{I}} V_{i}\right\} .
\end{aligned}
$$

It suffices to prove that $E\left(T_{3}^{2}\right) \underset{t \rightarrow \infty}{\longrightarrow} 0$ and $E\left(T_{4}^{2}\right) \underset{t \rightarrow \infty}{\longrightarrow} 0$. We begin with $T_{3}$, for which we obtain

$$
E\left(T_{3}^{2}\right)=f_{I}^{2}\left(\frac{1}{n_{I}}-\frac{1}{N_{I}}\right) S_{V, U_{I}}^{2}+\frac{f_{I}}{N_{I}}\left\{\frac{1}{N_{I}} \sum_{u_{i} \in U_{I}} V\left(\hat{V}_{i}\right)\right\},
$$


where $S_{V, U_{I}}^{2}=\left(N_{I}-1\right)^{-1} \sum_{u_{i} \in U_{I}}\left(V_{i}-N_{I}^{-1} \sum_{u_{j} \in U_{I}} V_{j}\right)^{2}$. From assumptions (H2) and (H3), there exists some constant $C_{7}$ such that $E\left(T_{3}^{2}\right) \leq C_{7} n_{I}^{-1}$, so that $E\left(T_{3}^{2}\right) \underset{t \rightarrow \infty}{\longrightarrow} 0$. We now consider $T_{4}$, for which $E\left(T_{4}\right)=0$ and $E\left(T_{4}^{2}\right)=$ $V\left(T_{4}\right)=\left(1-f_{I}\right)^{2} V\left(s_{Z}^{2}\right)$. We have

$$
V\left(s_{Z}^{2}\right)=V E_{\left\{S_{i}\right\}}\left(s_{Z}^{2}\right)+E V_{\left\{S_{i}\right\}}\left(s_{Z}^{2}\right)
$$

where we note $E_{\left\{S_{i}\right\}}$ and $V_{\left\{S_{i}\right\}}$ for the expectation and variance conditionally on the second-stage samples $S_{i}$ for $u_{i} \in U_{I}$. Under (H2), we obtain that there exists some constant $C_{8}$ such that $V E_{\left\{S_{i}\right\}}\left(s_{Z}^{2}\right) \leq C_{8} N_{I}^{-1}$, and some constant $C_{9}$ such that $E V_{\left\{S_{i}\right\}}\left(s_{Z}^{2}\right) \leq C_{9} n_{I}^{-1}$. Using (A8), we obtain that $E\left(T_{4}^{2}\right) \underset{t \rightarrow \infty}{\longrightarrow} 0$.

\subsection{Proof of Proposition 4.1.}

LEMMA 1.2. For any $u_{i} \neq u_{j} \in S_{I}^{d}$ :

$$
\begin{aligned}
E_{\left\{S_{I}^{d}\right\}}\left(W_{i}\right) & =\frac{n_{I}}{n_{I}^{d}}, \\
\operatorname{Cov}_{\left\{S_{I}^{d}\right\}}\left(W_{i}, W_{j}\right) & =-\frac{1}{n_{I}^{d}-1} V_{\left\{S_{I}^{d}\right\}}\left(W_{i}\right), \\
V_{\left\{S_{I}^{d}\right\}}\left(W_{i}\right) & =\frac{n_{I}}{\left(n_{I}^{d}\right)^{2}}\left\{\left(n_{I}^{d}-n_{I}\right)\right. \\
& \left.+n_{I}^{d}\left(n_{I}-1\right) \frac{S t\left(n_{I}-1, n_{I}^{d}\right)}{S t\left(n_{I}, n_{I}^{d}\right)}\right\}, \\
V_{\left\{S_{I}^{d}\right\}}\left(\sum_{u_{i} \in S_{I}^{d}} W_{i} Y_{i}\right) & =n_{I}^{d} S_{Y, S_{I}^{d}}^{2} V_{\left\{S_{I}^{d}\right\}}\left(W_{i}\right),
\end{aligned}
$$

with $S t(\cdot, \cdot)$ the Stirling number of the second kind.

Proof . We have $\sum_{u_{i} \in S_{I d}} W_{i}=n_{I}$, and for reasons of symmetry, equations (A9) and (A10) follow. With our notation, equation (11.5) in [3] reads as follows:

$$
\begin{aligned}
V_{\left\{S_{I}^{d}\right\}}\left(W_{i}\right) & =\frac{n_{I}}{\left(n_{I}^{d}\right)^{2}}\left\{\left(n_{I}^{d}-1\right)\right. \\
& \left.-\left(n_{I}-1\right) \frac{S t\left(n_{I}-1, n_{I}^{d}-1\right)}{S t\left(n_{I}, n_{I}^{d}\right)}\right\}
\end{aligned}
$$


From the identity $S t\left(n_{I}, n_{I}^{d}\right)=n_{I}^{d} S t\left(n_{I}-1, n_{I}^{d}\right)+S t\left(n_{I}-1, n_{I}^{d}-1\right)$, we obtain equation (A11). Finally, from $\sum_{u_{i} \in S_{I d}} W_{i}=n_{I}$, we obtain

$$
\begin{aligned}
V_{\left\{S_{I}^{d}\right\}}\left(\sum_{u_{i} \in S_{I}^{d}} W_{i} Y_{i}\right) & =-\frac{1}{2} \sum_{u_{i} \neq u_{j} \in S_{I}^{d}} \operatorname{Cov}_{\left\{S_{I}^{d}\right\}}\left(W_{i}, W_{j}\right)\left(Y_{i}-Y_{j}\right)^{2} \\
& =\left\{\frac{1}{2\left(n_{I}^{d}-1\right)} V_{\left\{S_{I}^{d}\right\}}\left(W_{i}\right)\right\} \sum_{u_{i} \neq u_{j} \in S_{I}^{d}}\left(Y_{i}-Y_{j}\right)^{2} \\
& =n_{I}^{d} S_{Y, S_{I}^{d}}^{2} V_{\left\{S_{I}^{d}\right\}}\left(W_{i}\right) .
\end{aligned}
$$

Lemma 1.3. Assume that the samples $S_{I}^{W R}$ and $S_{I}$ are selected according to Algorithm 2. Then

$$
\begin{aligned}
E_{\left\{n_{I}^{d}\right\}}\left(\hat{Y}_{W R}-\hat{Y}\right)^{2} & =\frac{N_{I}^{2}}{n_{I}}\left\{\left(n_{I}-1\right) \frac{S t\left(n_{I}-1, n_{I}^{d}\right)}{S t\left(n_{I}, n_{I}^{d}\right)} S_{Y, U_{I}}^{2}\right. \\
& \left.+2 \frac{n_{I}-n_{I}^{d}}{n_{I} N_{I}} \sum_{u_{i} \in U_{I}} V_{i}\right\}
\end{aligned}
$$

Proof . Since $S_{I}^{W R}$ and $S_{I}$ are independent of $n_{I}^{d}$, we have $E_{\left\{n_{I}^{d}\right\}}\left(\hat{Y}_{W R}-\right.$ $\hat{Y})=E\left(\hat{Y}_{W R}-\hat{Y}\right)=0$, so that $E_{\left\{n_{I}^{d}\right\}}\left(\hat{Y}_{W R}-\hat{Y}\right)^{2}=V_{\left\{n_{I}^{d}\right\}}\left(\hat{Y}_{W R}-\hat{Y}\right)$. Also, we have

$$
V_{\left\{n_{I}^{d}\right\}}\left(\hat{Y}_{W R}-\hat{Y}\right)=A_{1}+A_{2}+A_{3}+A_{4}
$$

where

$$
\begin{aligned}
& A_{1}=V_{\left\{n_{I}^{d}\right\}} E_{\left\{n_{I}^{d}, S_{I}^{d}\right\}}\left(\hat{Y}_{W R}-\hat{Y}\right) \\
& A_{2}=E_{\left\{n_{I}^{d}\right\}} V_{\left\{n_{I}^{d}, S_{I}^{d}\right\}} E_{\left\{n_{I}^{d}, S_{I}^{d}, W\right\}}\left(\hat{Y}_{W R}-\hat{Y}\right) \\
& A_{3}=E_{\left\{n_{I}^{d}\right\}} V_{\left\{n_{I}^{d}, S_{I}^{d}, W\right\}} E_{\left\{n_{I}^{d}, S_{I}^{d}, W, S_{I}^{c}\right\}}\left(\hat{Y}_{W R}-\hat{Y}\right) \\
& A_{4}=E_{\left\{n_{I}^{d}\right\}} V_{\left\{n_{I}^{d}, S_{I}^{d}, W, S_{I}^{c}\right\}}\left(\hat{Y}_{W R}-\hat{Y}\right) .
\end{aligned}
$$

We compute the four terms in (A15) separately, beginning with $A_{3}$. Since the difference between $\hat{Y}_{W R}$ and $\hat{Y}$ may be written as

$$
\hat{Y}_{W R}-\hat{Y}=\frac{N_{I}}{n_{I}}\left(\sum_{\substack{u_{i} \in S_{I}^{d} \\ W_{i}>1}} \sum_{j=2}^{W_{i}} \hat{Y}_{i[j]}-\sum_{u_{i} \in S_{I}^{c}} \hat{Y}_{i}\right),
$$

imsart-aos ver. 2014/10/16 file: SupplementaryMaterial_Rev2.tex date: May 17, 2015 
SUPPLEMENT TO COUPLING METHODS FOR MULTISTAGE SAMPLING 5 we obtain successively

$$
\begin{array}{r}
E_{\left\{n_{I}^{d}, S_{I}^{d}, W, S_{I}^{c}\right\}}\left(\hat{Y}_{W R}-\hat{Y}\right) \\
=\frac{N_{I}}{n_{I}}\left\{\sum_{u_{i} \in S_{I}^{d}}\left(W_{i}-1\right) Y_{i}-\sum_{u_{i} \in S_{I}^{c}} Y_{i}\right\},
\end{array}
$$

and

$$
\begin{array}{r}
V_{\left\{n_{I}^{d}, S_{I}^{d}, W\right\}} E_{\left\{n_{I}^{d}, S_{I}^{d}, W, S_{I}^{c}\right\}}\left(\tilde{Y}_{W R}-\hat{Y}\right) \\
=\frac{N_{I}^{2}}{n_{I}^{2}}\left(n_{I}-n_{I}^{d}\right)^{2}\left(\frac{1}{n_{I}-n_{I}^{d}}-\frac{1}{N_{I}-n_{I}^{d}}\right) S_{Y, U_{I} \backslash S_{I}^{d}}^{2}
\end{array}
$$

where $S_{Y, U_{I} \backslash S_{I}^{d}}^{2}=\left(N_{I}-n_{I}^{d}-1\right)^{-1} \sum_{u_{i} \in U_{I} \backslash S_{I}^{d}}\left(Y_{i}-\left(N_{I}-n_{I}^{d}\right)^{-1} \sum_{u_{j} \in U_{I}-S_{I}^{d}} Y_{j}\right)^{2}$.

Finally,

$$
A_{3}=\frac{N_{I}^{2}}{n_{I}^{2}} \frac{\left(N_{I}-n_{I}\right)\left(n_{I}-n_{I}^{d}\right)}{N_{I}-n_{I}^{d}} S_{Y, U_{I}}^{2}
$$

We now turn to $A_{2}$. From (A21), we have

$$
\begin{aligned}
E_{\left\{n_{I}^{d}, S_{I}^{d}, W\right\}}\left(\hat{Y}_{W R}-\hat{Y}\right) & =\frac{N_{I}}{n_{I}}\left\{\sum_{u_{i} \in S_{I}^{d}}\left(W_{i}-1\right) Y_{i}\right. \\
& \left.-\frac{n_{I}-n_{I}^{d}}{N_{I}-n_{I}^{d}} \sum_{u_{i} \in U_{I} \backslash S_{I}^{d}} Y_{i}\right\},
\end{aligned}
$$

so that

$$
V_{\left\{n_{I}^{d}, S_{I}^{d}\right\}} E_{\left\{n_{I}^{d}, S_{I}^{d}, W\right\}}\left(\hat{Y}_{W R}-\hat{Y}\right)=\left(\frac{N_{I}}{n_{I}}\right)^{2} V_{\left\{n_{I}^{d}, S_{I}^{d}\right\}}\left(\sum_{u_{i} \in S_{I}^{d}} W_{i} Y_{i}\right) .
$$

From (A11) and (A12), we have

$V_{\left\{n_{I}^{d}, S_{I}^{d}\right\}}\left\{\sum_{u_{i} \in S_{I}^{d}} W_{i} Y_{i}\right\}=n_{I}^{d} S_{Y, S_{I}^{d}}^{2} \frac{n_{I}}{\left(n_{I}^{d}\right)^{2}}\left\{\left(n_{I}^{d}-n_{I}\right)+n_{I}^{d}\left(n_{I}-1\right) \frac{S t\left(n_{I}-1, n_{I}^{d}\right)}{S t\left(n_{I}, n_{I}^{d}\right)}\right\}$

so that

$$
A_{2}=\frac{N_{I}^{2}}{n_{I}} S_{Y, U_{I}}^{2}\left\{\left(n_{I}-1\right) \frac{S t\left(n_{I}-1, n_{I}^{d}\right)}{S t\left(n_{I}, n_{I}^{d}\right)}-\frac{n_{I}-n_{I}^{d}}{n_{I}^{d}}\right\}
$$

imsart-aos ver. 2014/10/16 file: SupplementaryMaterial_Rev2.tex date: May 17, 2015 
We now turn to $A_{1}$. From (A23) and (A9), we obtain

$$
\begin{aligned}
E_{\left\{n_{I}^{d}, S_{I}^{d}\right\}}\left(\hat{Y}_{W R}-\hat{Y}\right) & =\frac{N_{I}}{n_{I}}\left\{\frac{n_{I}-n_{I}^{d}}{n_{I}^{d}} \sum_{u_{i} \in S_{I}^{d}} Y_{i}-\frac{n_{I}-n_{I}^{d}}{N_{I}-n_{I}^{d}} \sum_{u_{i} \in U_{I} \backslash S_{I}^{d}} Y_{i}\right\} \\
& =\frac{N_{I}}{n_{I}}\left\{\frac{N_{I}\left(n_{I}-n_{I}^{d}\right)}{n_{I}^{d}\left(N_{I}-n_{I}^{d}\right)} \sum_{u_{i} \in S_{I}^{d}} Y_{i}-\frac{n_{I}-n_{I}^{d}}{N_{I}-n_{I}^{d}} \sum_{u_{i} \in U_{I}} Y_{i}\right\}
\end{aligned}
$$

and

$$
A_{1}=\frac{N_{I}^{2}}{n_{I}} S_{Y, U_{I}}^{2} \frac{N_{I}\left(n_{I}-n_{I}^{d}\right)^{2}}{n_{I} n_{I}^{d}\left(N_{I}-n_{I}^{d}\right)} .
$$

Finally, we consider $A_{4}$. From (A20), we obtain successively

$$
\begin{aligned}
& V_{\left\{n_{I}^{d}, S_{I}^{d}, W, S_{I}^{c}\right\}}\left(\hat{Y}_{W R}-\hat{Y}\right)=\left(\frac{N_{I}}{n_{I}}\right)^{2}\left\{\sum_{u_{i} \in S_{I}^{d}}\left(W_{i}-1\right) V_{i}+\sum_{u_{i} \in S_{I}^{c}} V_{i}\right\}, \\
& E_{\left\{n_{I}^{d}, S_{I}^{d}, W\right\} V_{\left\{n_{I}^{d}, S_{I}^{d}, W, S_{I}^{c}\right\}}\left(\hat{Y}_{W R}-\hat{Y}\right)=}\left(\frac{N_{I}}{n_{I}}\right)^{2}\left\{\sum_{u_{i} \in S_{I}^{d}}\left(W_{i}-1\right) V_{i}\right. \\
&+\left.\frac{n_{I}-n_{I}^{d}}{N_{I}-n_{I}^{d}} \sum_{u_{i} \in U_{I} \backslash S_{I}^{d}} V_{i}\right\}, \\
&=\left(\frac{N_{I}}{n_{I}}\right)^{2}\left\{\frac{n_{I}-n_{I}^{d}}{n_{I}^{d}} \sum_{u_{i} \in S_{I}^{d}} V_{i}\right. \\
& E_{\left\{n_{I}^{d}, S_{I}^{d}\right\}} V_{\left\{n_{I}^{d}, S_{I}^{d}, W, S_{I}^{c}\right\}}\left(\hat{Y}_{W R}-\hat{Y}\right) \\
&+\left.\frac{n_{I}-n_{I}^{d}}{N_{I}-n_{I}^{d}} \sum_{u_{i} \in U_{I} \backslash S_{I}^{d}} V_{i}\right\},
\end{aligned}
$$

and

$$
A_{4}=2 \frac{N_{I}^{2}}{n_{I}^{2}} \frac{n_{I}-n_{I}^{d}}{N_{I}} \sum_{u_{i} \in U_{I}} V_{i}
$$

Plugging (A22), (A24), (A25) and (A26) into (A15) and using some straightforward algebra, we obtain (A14).

imsart-aos ver. 2014/10/16 file: SupplementaryMaterial_Rev2.tex date: May 17, 2015 
Using the probability distribution of $n_{I}^{d}$ (see for example [1], equation 10.48 , p. 443), we obtain

$$
\begin{aligned}
E\left\{\frac{S t\left(n_{I}-1, n_{I}^{d}\right)}{S t\left(n_{I}, n_{I}^{d}\right)}\right\} & =\sum_{k=1}^{n_{I}} \frac{N_{I} !}{\left(N_{I}-k\right) ! N_{I}^{n_{I}}} S t\left(n_{I}-1, k\right) \\
& =\frac{1}{N_{I}} \sum_{k=1}^{n_{I}-1} \frac{N_{I} !}{\left(N_{I}-k\right) ! N_{I}^{n_{I}-1}} S t\left(n_{I}-1, k\right) \\
& =\frac{1}{N_{I}} .
\end{aligned}
$$

Also, $E\left(n_{I}^{d}\right)=N_{I}\left\{1-\left(1-\frac{1}{N_{I}}\right)^{n_{I}}\right\}$, and from the inequality $(1-x)^{n_{I}} \leq$ $1-n_{I} x+\frac{n_{I}\left(n_{I}-1\right)}{2} x^{2}$ which is true for $0 \leq x \leq 1$, we obtain

$$
E\left(\frac{n_{I}-n_{I}^{d}}{n_{I}}\right) \leq \frac{n_{I}-1}{2 N_{I}} \leq \frac{n_{I}-1}{2\left(N_{I}-1\right)}
$$

From (A14), (A27) and (A28), we obtain

$$
E\left(\hat{Y}_{W R}-\hat{Y}\right)^{2} \leq \frac{N_{I}^{2}}{n_{I}}\left\{\frac{n_{I}-1}{N_{I}} S_{Y, U_{I}}^{2}+\frac{n_{I}-1}{N_{I}-1} \frac{1}{N_{I}} \sum_{u_{i} \in U_{I}} V_{i}\right\}
$$

and using (4.3), we obtain (4.8).

1.5. Proof of Proposition 4.2. From (4.8), equation (4.9) is immediate. Turning to (4.10), we may rewrite

$$
s_{Z}^{2}=\frac{1}{n_{I}-1} \sum_{u_{i} \in S_{I}}\left(\hat{Y}_{i}-\bar{X}\right)^{2}-\frac{n_{I}}{n_{I}-1}(\bar{Z}-\bar{X})^{2} .
$$

From (4.9), it is sufficient to prove that $E\left(\left|A_{5}\right|\right) \underset{t \rightarrow \infty}{\longrightarrow} 0$ where

$$
A_{5} \equiv \frac{1}{n_{I}-1} \sum_{u_{i} \in S_{I}}\left(\hat{Y}_{i}-\bar{X}\right)^{2}-s_{X}^{2}
$$


We have

$$
\begin{aligned}
\left|A_{5}\right| & =\left|\frac{1}{n_{I}-1} \sum_{u_{i} \in S_{I}^{c}}\left(\hat{Y}_{i}-\frac{\hat{Y}_{W R}}{N_{I}}\right)^{2}-\frac{1}{n_{I}-1} \sum_{\substack{u_{i} \in S_{I}^{d} \\
W_{i}>1}}^{W_{j}}\left(\hat{Y}_{i[j]}-\frac{\hat{Y}_{W R}}{N_{I}}\right)^{2}\right| \\
& \leq \frac{2}{n_{I}-1} \sum_{u_{i} \in S_{I}^{c}}\left(\hat{Y}_{i}^{2}+\frac{\hat{Y}_{W R}^{2}}{N_{I}^{2}}\right)+\frac{2}{n_{I}-1} \sum_{u_{i} \in S_{I}^{d}} \sum_{j=2}^{W_{i}}\left(\hat{Y}_{i[j]}^{2}+\frac{\hat{Y}_{W R}^{2}}{N_{I}^{2}}\right) \\
& =\frac{2}{n_{I}-1} \sum_{u_{i} \in S_{I}^{c}} \hat{Y}_{i}^{2}+\frac{2}{n_{I}-1} \sum_{u_{i} \in S_{I}^{d}} \sum_{W_{i}>1}^{W_{i}} \hat{Y}_{i[j]}^{2}+\frac{4\left(n_{I}-n_{I}^{d}\right)}{n_{I}-1} \frac{\hat{Y}_{W R}^{2}}{N_{I}^{2}} .
\end{aligned}
$$

From (H2), there exists some constant $C_{11}$ such that $E_{\left\{n_{I}^{d}\right\}}\left|A_{5}\right| \leq C_{11}\left(n_{I}-\right.$ $1)^{-1}\left(n_{I}-n_{I}^{d}\right)$, and from equation (A28), $E\left|A_{5}\right| \underset{t \rightarrow \infty}{\longrightarrow} 0$.

1.6. Proof of Proposition 4.3. We may write

$$
E\left(\bar{Z}_{m}^{*}-\bar{X}_{m}^{*}\right)^{2}=E\left\{E_{\{Z, X\}}\left(\bar{Z}_{m}^{*}-\bar{X}_{m}^{*}\right)^{2}\right\}
$$

where we note $E_{\{Z, X\}}(\cdot)$ and $V_{\{Z, X\}}(\cdot)$ for the expectation and variance conditionally on $Z_{1}, \ldots, Z_{n_{I}}$ and $X_{1}, \ldots, X_{n_{I}}$. We have

$$
\begin{aligned}
E_{\{Z, X\}}\left(\bar{Z}_{m}^{*}-\bar{X}_{m}^{*}\right)^{2} & =E_{\{Z, X\}}\left(\frac{1}{m} \sum_{j=1}^{n_{I}} D_{j}\left(Z_{j}-X_{j}\right)\right)^{2} \\
& =\frac{1}{m n_{I}} \sum_{j=1}^{n_{I}}\left(X_{j}-Z_{j}-\bar{X}+\bar{Z}\right)^{2} \\
& +\left\{\frac{1}{n_{I}} \sum_{j=1}^{n_{I}}\left(Z_{j}-X_{j}\right)\right\}^{2} \\
& =\frac{1}{m n_{I}} \sum_{j=1}^{n_{I}}\left(X_{j}-Z_{j}\right)^{2}+\frac{m-1}{m}(\bar{X}-\bar{Z})^{2} .
\end{aligned}
$$

which leads to

$$
\begin{aligned}
E\left(\bar{Z}_{m}^{*}-\bar{X}_{m}^{*}\right)^{2} & =\frac{1}{m} E\left\{\frac{1}{n_{I}} \sum_{j=1}^{n_{I}}\left(X_{j}-Z_{j}\right)^{2}\right\} \\
& +\frac{m-1}{m} E(\bar{X}-\bar{Z})^{2} .
\end{aligned}
$$

imsart-aos ver. 2014/10/16 file: SupplementaryMaterial_Rev2.tex date: May 17, 2015 
We consider the first term in the right-hand side of (A30) first. We have

$$
\begin{aligned}
& \frac{1}{n_{I}} \sum_{j=1}^{n_{I}}\left(Z_{j}-X_{j}\right)^{2} \leq \frac{2}{n_{I}} \sum_{j ; Z_{j} \neq X_{j}}\left(Z_{j}^{2}+X_{j}^{2}\right) \\
& =\frac{2}{n_{I}}\left[\sum_{u_{i} \in S_{I}^{c}}\left(\hat{Y}_{i}\right)^{2}+\sum_{\substack{u_{i} \in S_{I}^{d} \\
W_{i}>1}} \sum_{j=2}^{W_{i}}\left(\hat{Y}_{i[j]}\right)^{2}\right] .
\end{aligned}
$$

Using a similar reasoning as in the proof of Proposition 1.3, we obtain

$$
\begin{aligned}
E\left[\frac{2}{n_{I}}\left\{\sum_{u_{i} \in S_{I}^{c}}\left(\hat{Y}_{i}\right)^{2}+\sum_{\substack{u_{i} \in S_{I}^{d} \\
W_{i}>1}} \sum_{j=2}^{W_{i}}\left(\hat{Y}_{i[j]}\right)^{2}\right\}\right] & =2 E\left[\frac{n_{I}-n_{I}^{d}}{n_{I} \times N_{I}} \sum_{u_{i} \in U_{I}}\left(Y_{i}^{2}+V_{i}\right)\right] \\
& \leq 2 C_{12} E\left(\frac{n_{I}-n_{I}^{d}}{n_{I}}\right) \\
& \leq C_{12} \frac{n_{I}-1}{N_{I}-1}
\end{aligned}
$$

for some constant $C_{12}$, where the last line follows from (A28). It follows that

$$
E\left\{\frac{1}{n_{I}} \sum_{j=1}^{n_{I}}\left(Z_{j}-X_{j}\right)^{2}\right\}=o(1)
$$

and the first term in the right-hand side of (A30) is $o\left(m^{-1}\right)$. From (4.9), the second term in the right-hand side of (A30) is $o\left(n_{I}^{-1}\right)$, which completes the proof.

1.7. Proof of Proposition 4.6. We can write

$$
\frac{V_{\{Z\}}\left(\hat{\theta}^{*}\right)}{V(\hat{\theta})}=\frac{V\left(\hat{\theta}_{W R}\right)}{V(\hat{\theta})}\left(A_{9}+A_{10}\right)
$$

where

$$
A_{9}=\frac{V_{\{X\}}\left(\hat{\theta}_{W R}^{*}\right)}{V\left(\hat{\theta}_{W R}\right)} \quad \text { and } \quad A_{10}=\frac{V_{\{Z\}}\left(\hat{\theta}^{*}\right)-V_{\{X\}}\left(\hat{\theta}_{W R}^{*}\right)}{V\left(\hat{\theta}_{W R}\right)} .
$$

Making use of the inequality

$$
\left\{\{V(\hat{\theta})\}^{0.5}-\left\{V\left(\hat{\theta}_{W R}\right)\right\}^{0.5}\right\}^{2} \leq E\left(\hat{\theta}-\hat{\theta}_{W R}\right)^{2}
$$

imsart-aos ver. 2014/10/16 file: SupplementaryMaterial_Rev2.tex date: May 17, 2015 
we obtain by equation (4.20) and by (H6) that $\{V(\hat{\theta})\}^{-1} V\left(\hat{\theta}_{W R}\right) \underset{t \rightarrow \infty}{\longrightarrow} 1$. Similarly, making use of the inequality

$$
\begin{aligned}
E\left\{\left\{V_{\{Z\}}\left(\hat{\theta}^{*}\right)\right\}^{0.5}-\left\{V_{\{X\}}\left(\hat{\theta}_{W R}^{*}\right)\right\}^{0.5}\right\}^{2} & \leq E\left\{E_{\{Z, X\}}\left(\hat{\theta}^{*}-\hat{\theta}_{W R}^{*}\right)^{2}\right\} \\
& =E\left(\hat{\theta}^{*}-\hat{\theta}_{W R}^{*}\right)^{2}
\end{aligned}
$$

we obtain by equation (4.22) and by (H6) that $E\left\{\left(A_{10}\right)^{2}\right\}=o(1)$, so that $A_{10} \underset{P r}{\longrightarrow}$ 0. From (A32), the result follows.

2. Additional simulation results. In Section 5 of the article, we described the set-up of a simulation study to investigate on the performance of the variance estimators. Three finite populations with $N_{I}=2,000$ PSUs were generated. The simulation results for Population 3 were given in Tables 1 and 2 of the paper. In this Section, we present the simulation results obtained for Population 1, which are given in Tables A1 and A2, and the simulation results obtained for Population 2, which are given in Tables A3 and $\mathrm{A} 4$

\title{
References.
}

[1] Johnson, N.L., Kemp, A.W. And Kotz, S. (2005). Univariate discrete distributions, 3rd ed. Wiley, New York. MR2163227

[2] Wooff, D.A. (1985). Bounds on reciprocal moments with applications and developments in Stein estimation and post-stratification. J. R. Stat. Soc. Ser. B Stat. Methodol. 47 362-371. MR0816102

[3] Nandi, S.B. And Dutta, S.K. (1986). Some discrete distributions involving Stirling numbers. Sankhya Ser. B 48 301-314. MR0905207

\author{
Rue Blaise Pascal \\ Campus de Ker LanN \\ 35170 BRUZ \\ FRANCE \\ E-MAIL: chauvet@ensai.fr
}


TABle A1

Relative Bias, Relative Stability And Nominal One-Tailed Error Rates For The Simplified Variance Estimator Of The Horvitz-Thompson Estimator, And For The Bootstrap For The Estimation Of A Total For Population 1

\begin{tabular}{|c|c|c|c|c|c|c|c|c|c|}
\hline & \multirow{3}{*}{$\begin{array}{l}n_{0} \\
n_{I}\end{array}$} & \multicolumn{8}{|c|}{ Simplified Variance Estimator $v_{S I M P}(\hat{Y})$ For $Y_{2 h-1}$} \\
\hline & & \multicolumn{4}{|c|}{5} & \multicolumn{4}{|c|}{10} \\
\hline & & 20 & 40 & 100 & 200 & 20 & 40 & 100 & 200 \\
\hline \multirow[t]{5}{*}{$\rho=0.1$} & $\mathrm{RB}$ & -0.01 & -0.01 & -0.04 & -0.07 & -0.01 & 0.00 & -0.04 & -0.05 \\
\hline & $\mathrm{RS}$ & 0.31 & 0.22 & 0.14 & 0.12 & 0.32 & 0.22 & 0.14 & 0.11 \\
\hline & $\mathrm{L}$ & 3.7 & 3.7 & 2.4 & 2.7 & 4.7 & 3.5 & 3.0 & 3.0 \\
\hline & $\mathrm{U}$ & 3.7 & 2.0 & 3.4 & 3.5 & 3.0 & 3.0 & 3.3 & 2.4 \\
\hline & $\mathrm{L}+\mathrm{U}$ & 7.4 & 5.7 & 5.8 & 6.2 & 7.7 & 6.5 & 6.3 & 5.4 \\
\hline \multirow[t]{5}{*}{$\rho=0.2$} & $\mathrm{RB}$ & -0.02 & -0.02 & -0.03 & -0.06 & 0.00 & 0.00 & -0.03 & -0.04 \\
\hline & $\mathrm{RS}$ & 0.32 & 0.22 & 0.14 & 0.11 & 0.32 & 0.21 & 0.14 & 0.10 \\
\hline & $\mathrm{L}$ & 3.6 & 3.6 & 4.4 & 2.6 & 4.0 & 3.6 & 3.4 & 3.2 \\
\hline & $\mathrm{U}$ & 2.6 & 2.8 & 3.9 & 2.9 & 2.6 & 3.6 & 2.6 & 2.5 \\
\hline & $\mathrm{L}+\mathrm{U}$ & 6.2 & 6.4 & 8.3 & 5.5 & 6.6 & 7.2 & 6.0 & 5.7 \\
\hline \multirow[t]{8}{*}{$\rho=0.3$} & $\mathrm{RB}$ & -0.01 & -0.01 & -0.01 & -0.03 & 0.00 & 0.01 & -0.02 & -0.01 \\
\hline & $\mathrm{RS}$ & 0.33 & 0.22 & 0.14 & 0.10 & 0.33 & 0.23 & 0.14 & 0.10 \\
\hline & $\mathrm{L}$ & 3.2 & 3.7 & 2.2 & 3.0 & 3.6 & 4.0 & 3.4 & 3.0 \\
\hline & $\mathrm{U}$ & 2.8 & 3.1 & 4.0 & 3.2 & 3.3 & 2.8 & 3.5 & 3.5 \\
\hline & $\mathrm{L}+\mathrm{U}$ & 6.0 & 6.8 & 6.2 & 6.2 & 6.9 & 6.8 & 6.9 & 6.5 \\
\hline & & \multicolumn{8}{|c|}{ Bootstrap of PSUs For $Y_{2 h-1}$} \\
\hline & $n_{0}$ & \multicolumn{4}{|c|}{5} & \multicolumn{4}{|c|}{10} \\
\hline & $n_{I}$ & 20 & 40 & 100 & 200 & 20 & 40 & 100 & 200 \\
\hline \multirow[t]{5}{*}{$\rho=0.1$} & $\mathrm{RB}$ & -0.01 & 0.01 & 0.02 & 0.03 & 0.01 & 0.02 & 0.01 & 0.06 \\
\hline & $\mathrm{RS}$ & 0.32 & 0.23 & 0.15 & 0.12 & 0.33 & 0.23 & 0.15 & 0.13 \\
\hline & $\mathrm{L}$ & 4.0 & 3.8 & 2.2 & 2.0 & 4.7 & 3.2 & 2.8 & 2.3 \\
\hline & $\mathrm{U}$ & 3.5 & 2.1 & 3.3 & 2.8 & 3.3 & 2.8 & 2.5 & 1.6 \\
\hline & $\mathrm{L}+\mathrm{U}$ & 7.5 & 5.9 & 5.5 & 4.8 & 8.0 & 6.0 & 5.3 & 3.9 \\
\hline \multirow[t]{5}{*}{$\rho=0.2$} & $\mathrm{RB}$ & -0.02 & 0.00 & 0.02 & 0.04 & 0.01 & 0.02 & 0.03 & 0.07 \\
\hline & $\mathrm{RS}$ & 0.32 & 0.23 & 0.16 & 0.12 & 0.33 & 0.22 & 0.15 & 0.14 \\
\hline & $\mathrm{L}$ & 3.7 & 3.7 & 4.4 & 2.4 & 3.9 & 3.5 & 3.4 & 2.1 \\
\hline & $\mathrm{U}$ & 2.5 & 2.6 & 3.8 & 2.0 & 2.7 & 3.3 & 2.4 & 2.1 \\
\hline & $\mathrm{L}+\mathrm{U}$ & 6.2 & 6.3 & 8.2 & 4.4 & 6.6 & 6.8 & 5.8 & 4.2 \\
\hline \multirow[t]{5}{*}{$\rho=0.3$} & $\mathrm{RB}$ & -0.01 & 0.01 & 0.04 & 0.08 & 0.01 & 0.03 & 0.03 & 0.10 \\
\hline & $\mathrm{RS}$ & 0.33 & 0.23 & 0.17 & 0.14 & 0.33 & 0.24 & 0.16 & 0.16 \\
\hline & $\mathrm{L}$ & 3.1 & 3.5 & 2.0 & 2.7 & 3.5 & 3.7 & 3.1 & 2.5 \\
\hline & $\mathrm{U}$ & 2.8 & 3.1 & 4.0 & 3.0 & 3.3 & 2.6 & 3.0 & 2.9 \\
\hline & $\mathrm{L}+\mathrm{U}$ & 5.9 & 6.6 & 6.0 & 5.7 & 6.8 & 6.3 & 6.1 & 5.4 \\
\hline
\end{tabular}


TABLE A2

Relative Bias, Relative Stability And Nominal One-Tailed Error Rates For The Bootstrap For The Estimation Of A Ratio And A Coefficient Of Correlation For Population 1

\begin{tabular}{|c|c|c|c|c|c|c|c|c|c|}
\hline & \multirow{3}{*}{$\begin{array}{l}n_{0} \\
n_{I}\end{array}$} & \multicolumn{8}{|c|}{ Bootstrap of PSUs For $R_{h}$} \\
\hline & & \multicolumn{4}{|c|}{5} & \multicolumn{4}{|c|}{10} \\
\hline & & 20 & 40 & 100 & 200 & 20 & 40 & 100 & 200 \\
\hline \multirow[t]{5}{*}{$\rho=0.1$} & RB & 0.02 & 0.02 & 0.01 & 0.01 & 0.00 & 0.03 & 0.00 & 0.04 \\
\hline & $\mathrm{RS}$ & 0.34 & 0.24 & 0.15 & 0.11 & 0.33 & 0.24 & 0.16 & 0.12 \\
\hline & $\mathrm{L}$ & 3.6 & 2.8 & 2.8 & 3.0 & 3.9 & 3.2 & 3.3 & 2.3 \\
\hline & $\mathrm{U}$ & 2.9 & 3.3 & 2.2 & 3.0 & 3.1 & 2.8 & 2.4 & 2.7 \\
\hline & $\mathrm{L}+\mathrm{U}$ & 6.5 & 6.1 & 5.0 & 6.0 & 7.0 & 6.0 & 5.7 & 5.0 \\
\hline \multirow[t]{5}{*}{$\rho=0.2$} & $\mathrm{RB}$ & 0.00 & 0.02 & 0.02 & 0.02 & 0.00 & -0.02 & 0.02 & 0.02 \\
\hline & $\mathrm{RS}$ & 0.33 & 0.25 & 0.15 & 0.11 & 0.34 & 0.22 & 0.15 & 0.11 \\
\hline & $\mathrm{L}$ & 4.3 & 3.0 & 2.1 & 2.0 & 3.9 & 3.2 & 2.7 & 3.0 \\
\hline & $\mathrm{U}$ & 3.0 & 2.0 & 2.4 & 2.6 & 2.9 & 3.3 & 2.4 & 2.4 \\
\hline & $\mathrm{L}+\mathrm{U}$ & 7.3 & 5.0 & 4.5 & 4.6 & 6.8 & 6.5 & 5.1 & 5.4 \\
\hline \multirow[t]{8}{*}{$\rho=0.3$} & $\mathrm{RB}$ & 0.02 & 0.02 & 0.02 & 0.02 & 0.02 & 0.01 & 0.02 & 0.04 \\
\hline & $\mathrm{RS}$ & 0.35 & 0.23 & 0.15 & 0.11 & 0.34 & 0.24 & 0.16 & 0.12 \\
\hline & $\mathrm{L}$ & 3.1 & 3.2 & 1.5 & 3.6 & 3.2 & 3.1 & 1.9 & 1.8 \\
\hline & $\mathrm{U}$ & 2.8 & 3.1 & 2.3 & 2.5 & 4.2 & 3.5 & 2.3 & 3.7 \\
\hline & $\mathrm{L}+\mathrm{U}$ & 5.9 & 6.3 & 3.8 & 6.1 & 7.4 & 6.6 & 4.2 & 5.5 \\
\hline & & \multicolumn{8}{|c|}{ Bootstrap of PSUs For $r_{h}$} \\
\hline & $n_{0}$ & \multicolumn{4}{|c|}{5} & \multicolumn{4}{|c|}{10} \\
\hline & $n_{I}$ & 20 & 40 & 100 & 200 & 20 & 40 & 100 & 200 \\
\hline \multirow[t]{5}{*}{$\rho=0.1$} & $\mathrm{RB}$ & 0.01 & 0.00 & 0.02 & 0.01 & -0.01 & 0.01 & 0.05 & 0.03 \\
\hline & $\mathrm{RS}$ & 0.41 & 0.29 & 0.19 & 0.14 & 0.37 & 0.27 & 0.19 & 0.13 \\
\hline & $\mathrm{L}$ & 3.8 & 2.9 & 3.2 & 2.8 & 3.3 & 2.5 & 2.0 & 2.2 \\
\hline & $\mathrm{U}$ & 3.2 & 2.8 & 2.6 & 2.1 & 3.2 & 3.0 & 2.6 & 2.4 \\
\hline & $\mathrm{L}+\mathrm{U}$ & 7.0 & 5.7 & 5.8 & 4.9 & 6.5 & 5.5 & 4.6 & 4.6 \\
\hline \multirow[t]{5}{*}{$\rho=0.2$} & $\mathrm{RB}$ & 0.02 & 0.03 & -0.01 & 0.00 & 0.01 & -0.01 & 0.03 & 0.04 \\
\hline & $\mathrm{RS}$ & 0.44 & 0.30 & 0.19 & 0.13 & 0.39 & 0.26 & 0.18 & 0.13 \\
\hline & $\mathrm{L}$ & 3.2 & 2.4 & 2.9 & 2.6 & 2.7 & 3.2 & 1.4 & 2.7 \\
\hline & $\mathrm{U}$ & 3.1 & 2.5 & 1.9 & 2.9 & 3.8 & 2.9 & 3.2 & 1.8 \\
\hline & $\mathrm{L}+\mathrm{U}$ & 6.3 & 4.9 & 4.8 & 5.5 & 6.5 & 6.1 & 4.6 & 4.5 \\
\hline \multirow[t]{5}{*}{$\rho=0.3$} & RB & 0.02 & 0.00 & 0.02 & 0.02 & 0.02 & -0.01 & 0.03 & 0.03 \\
\hline & $\mathrm{RS}$ & 0.46 & 0.32 & 0.20 & 0.15 & 0.41 & 0.27 & 0.19 & 0.14 \\
\hline & $\mathrm{L}$ & 3.5 & 2.9 & 2.2 & 3.0 & 2.7 & 2.4 & 1.4 & 1.9 \\
\hline & $\mathrm{U}$ & 4.1 & 2.6 & 3.4 & 2.0 & 5.8 & 3.5 & 3.5 & 3.8 \\
\hline & $\mathrm{L}+\mathrm{U}$ & 7.6 & 5.5 & 5.6 & 5.0 & 8.5 & 5.9 & 4.9 & 5.7 \\
\hline
\end{tabular}


TABle A3

Relative Bias, Relative Stability And Nominal One-Tailed Error Rates For The Simplified Variance Estimator Of The Horvitz-Thompson Estimator, And For The Bootstrap For The Estimation Of A Total For Population 2

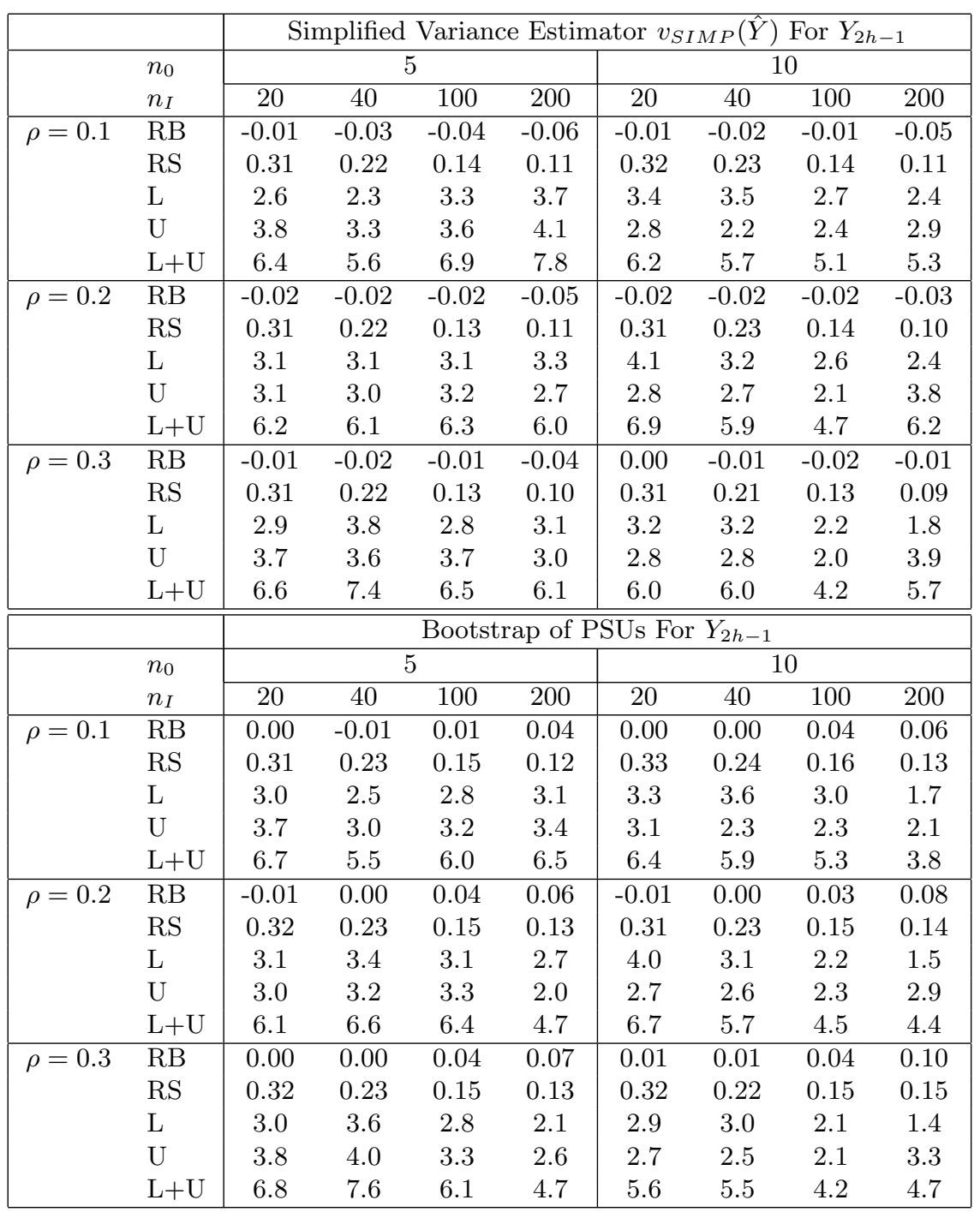


TABLE A4

Relative Bias, Relative Stability And Nominal One-Tailed Error Rates For The Bootstrap For The Estimation Of A Ratio And A Coefficient Of Correlation For Population 2

\begin{tabular}{|c|c|c|c|c|c|c|c|c|c|}
\hline & & & & Boot: & ap of & SUs I & r $R_{h}$ & & \\
\hline & $n_{0}$ & & & & & & & & \\
\hline & $n_{I}$ & 20 & 40 & 100 & 200 & 20 & 40 & 100 & 200 \\
\hline$\rho=0.1$ & $\mathrm{RB}$ & 0.00 & 0.00 & 0.00 & 0.02 & -0.01 & 0.00 & 0.02 & 0.02 \\
\hline & $\mathrm{RS}$ & 0.34 & 0.22 & 0.15 & 0.11 & 0.33 & 0.24 & 0.16 & 0.11 \\
\hline & $\mathrm{L}$ & 3.8 & 2.1 & 2.5 & 3.4 & 3.7 & 3.2 & 3.3 & 2.6 \\
\hline & $\mathrm{U}$ & 3.6 & 3.0 & 2.5 & 2.9 & 2.6 & 3.5 & 2.2 & 2.6 \\
\hline & $\mathrm{L}+\mathrm{U}$ & 7.4 & 5.1 & 5.0 & 6.3 & 6.3 & 6.7 & 5.5 & 5.2 \\
\hline$\rho=0.2$ & $\mathrm{RB}$ & -0.01 & 0.02 & -0.01 & 0.00 & 0.01 & 0.01 & 0.00 & 0.03 \\
\hline & $\mathrm{RS}$ & 0.34 & 0.24 & 0.15 & 0.11 & 0.32 & 0.23 & 0.16 & 0.12 \\
\hline & $\mathrm{L}$ & 3.3 & 3.4 & 3.0 & 2.6 & 3.2 & 2.9 & 2.0 & 2.2 \\
\hline & $\mathrm{U}$ & 2.8 & 3.1 & 2.6 & 1.6 & 3.7 & 3.0 & 2.4 & 2.1 \\
\hline & $\mathrm{L}+\mathrm{U}$ & 6.1 & 6.5 & 5.6 & 4.2 & 6.9 & 5.9 & 4.4 & 4.3 \\
\hline$\rho=0.3$ & $\mathrm{RB}$ & 0.01 & 0.00 & 0.01 & 0.01 & 0.02 & 0.03 & 0.01 & 0.03 \\
\hline & $\mathrm{RS}$ & 0.33 & 0.22 & 0.15 & 0.11 & 0.32 & 0.23 & 0.15 & 0.12 \\
\hline & $\mathrm{L}$ & 3.7 & 2.4 & 2.4 & 3.0 & 2.6 & 2.7 & 2.5 & 2.3 \\
\hline & $\mathrm{U}$ & 3.1 & 3.6 & 3.6 & 2.1 & 2.6 & 3.5 & 2.5 & 2.3 \\
\hline & $\mathrm{L}+\mathrm{U}$ & 6.8 & 6.0 & 6.0 & 5.1 & 5.2 & 6.2 & 5.0 & 4.6 \\
\hline & & & & Boot & ap of & SUs & $r r_{h}$ & & \\
\hline & $n_{0}$ & & & & & & & & \\
\hline & $n_{I}$ & 20 & 40 & 100 & 200 & 20 & 40 & 100 & 200 \\
\hline$\rho=0.1$ & $\mathrm{RB}$ & -0.01 & 0.02 & 0.02 & 0.01 & 0.01 & 0.00 & 0.02 & 0.04 \\
\hline & $\mathrm{RS}$ & 0.41 & 0.31 & 0.19 & 0.14 & 0.40 & 0.28 & 0.17 & 0.13 \\
\hline & $\mathrm{L}$ & 4.2 & 2.6 & 3.0 & 2.2 & 2.9 & 4.1 & 2.9 & 2.5 \\
\hline & $\mathrm{U}$ & 3.2 & 2.9 & 2.9 & 2.7 & 3.7 & 2.8 & 2.4 & 3.4 \\
\hline & $\mathrm{L}+\mathrm{U}$ & 7.4 & 5.5 & 5.9 & 4.9 & 6.6 & 6.9 & 5.3 & 5.9 \\
\hline$\rho=0.2$ & $\mathrm{RB}$ & 0.04 & 0.02 & 0.01 & 0.01 & 0.02 & 0.01 & 0.00 & 0.03 \\
\hline & $\mathrm{RS}$ & 0.44 & 0.30 & 0.19 & 0.14 & 0.40 & 0.29 & 0.17 & 0.13 \\
\hline & $\mathrm{L}$ & 4.0 & 2.6 & 2.4 & 1.8 & 3.0 & 1.6 & 2.4 & 2.2 \\
\hline & $\mathrm{U}$ & 3.7 & 3.5 & 2.7 & 3.1 & 4.4 & 3.5 & 2.9 & 2.0 \\
\hline & $\mathrm{L}+\mathrm{U}$ & 7.7 & 6.1 & 5.1 & 4.9 & 7.4 & 5.1 & 5.3 & 4.2 \\
\hline$\rho=0.3$ & $\mathrm{RB}$ & 0.00 & 0.00 & 0.01 & 0.01 & -0.04 & 0.02 & 0.01 & 0.04 \\
\hline & $\mathrm{RS}$ & 0.41 & 0.32 & 0.21 & 0.15 & 0.36 & 0.28 & 0.17 & 0.13 \\
\hline & $\mathrm{L}$ & 2.7 & 3.4 & 3.1 & 1.9 & 3.2 & 2.6 & 2.5 & 2.3 \\
\hline & $\mathrm{U}$ & 3.7 & 3.3 & 2.0 & 2.7 & 3.3 & 3.3 & 4.5 & 3.3 \\
\hline & $\mathrm{L}+\mathrm{U}$ & 6.4 & 6.7 & 5.1 & 4.6 & 6.5 & 5.9 & 7.0 & 5.6 \\
\hline
\end{tabular}

